\title{
青蓠素降解产物失碳倍半落内酯的全合成*
}

\author{
许杏样 吴照华 沈季铭 陈朝环 吴航林 周维善 \\ (中国科学院上海有机化学研究所)
}

青蓠素 1 是一个含有过氧基团的新型倍半萜内酯, 它具有突出的抗疮作用. 在测定结构 的基础上 ${ }^{[1]}$, 我们开展了它的全合成工作. 本文报道中间体失碳倍半萜内酯 10 的合成. 合成 的原料为 $10 \mathrm{R}(+)$ - 香草醛 2(见图 1). 2 用溴化锌环化立体专一地生成 $6 \mathrm{R}, 7 \mathrm{~S}, 10 \mathrm{R}(-$ )异胡薄荷醇 $3^{[2]} .3$ 依次用二嗍烷和过氧化氢处理得双差基物 4 , 推定其 $\mathrm{C}_{11}$-甲基为 $\beta$-构型 ${ }^{[3]}$. 4 用铬酸氧化得 $\boldsymbol{\gamma}$-内酯 5 ，IR： $\nu_{\max } 1787 \mathrm{~cm}^{-1}$ ( $\boldsymbol{\gamma}$-内酯). 5 用碱打开内酯, 然后经酯化和氧 化即得酮酯 6, $[\alpha]_{D}^{15}=-19^{\circ}\left(\mathrm{CHCl}_{3}, \mathrm{C}=1.45\right)$; IR： $\nu_{\max } 1710$ (酮), 1745 (酯) $\mathrm{cm}^{-1}$; $\operatorname{nmr}\left(\mathrm{CCl}_{4}\right): \delta: 0.98\left(3 \mathrm{H}, d, J=6 \mathrm{~Hz}, \mathrm{C}_{10}-\mathrm{CH}_{3}\right), 1.10\left(3 \mathrm{H}, d, J=6 \mathrm{~Hz}, \mathrm{C}_{11}-\mathrm{CH}_{3}\right)$, $3.56\left(3 \mathrm{H}, \mathrm{S},-\mathrm{COOCH}_{3}\right) \mathrm{ppm} ; \mathrm{ORD}\left(1.05 \%, \mathrm{CH}_{3} \mathrm{OH}\right): \Phi^{275}=-916, \Phi^{311}=-56$, $\Phi^{330}=-168, \Phi^{350}=-190, \Phi^{530}=-90$. 由于 6 的旋光色散呈现一个弱的、正的 Cotton 效 应,所以环上 $\mathrm{C}_{7}$-和 $\mathrm{C}_{10}$-取代基互为反式,并且均为横键 ${ }^{[4]} .4 \rightarrow 6$ 的总产率为 $50 \%$.

为了在 6 的 $\mathrm{C}_{1}$-位选择性地加人四个碳的边链, 我们首先在该位引进定向活化基团. 在 氢化钠的作用下, 6 和甲酸乙酯缩合 ${ }^{[s]}$, 同时伴有酯的交换,生成 $\beta$-醛酮 7,UV: $\lambda_{\mathrm{m} 2 \mathrm{x}}^{\mathrm{E} \text { toH }} 288.5 \mathrm{~nm}$ $(\epsilon 7100)$, 加碱 $\lambda_{\text {max }}^{\mathrm{EtOH}} 317 \mathrm{~nm}(\epsilon 16500) ; \mathrm{IR}: \nu_{\max } 1735$ (酯),1715 (肙、酮)、1635 和 1590 (宽. $\beta$-二酮) $\mathrm{cm}^{-1} ; \mathrm{nmr}\left(\mathrm{CCl}_{4}\right): \delta: 1-1.3\left(9 \mathrm{H}, \mathrm{m}, \mathrm{C}_{10}-\mathrm{CH}_{3} \mathrm{C}_{11}-\mathrm{CH}_{3},-\mathrm{OCH}_{2} \mathrm{CH}_{3}\right)$,
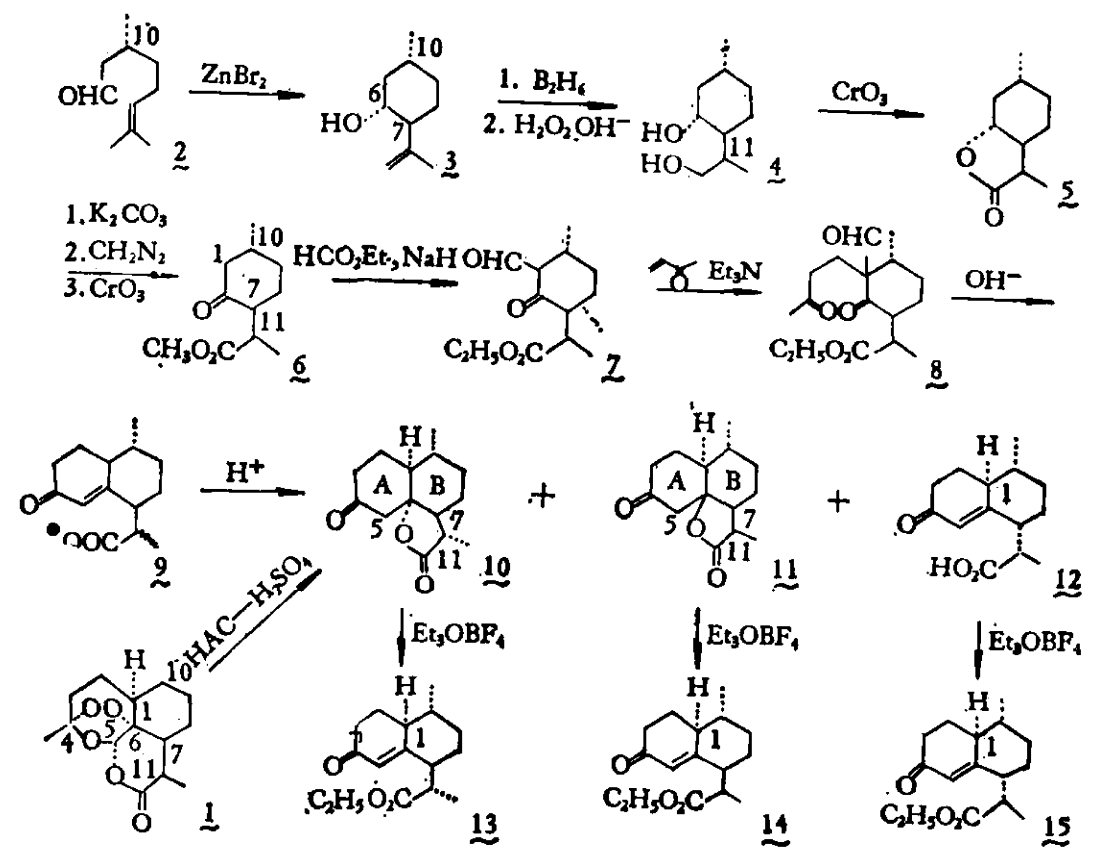

图 1 失碳倍半萜内蹜 10 的合成

* 参加本工作的还有朱杰同志。 
3.9-4.2 (2H, m, - $\left.\mathrm{OCH}_{2} \mathrm{CH}_{3}\right), 8.24$ 和 $8.4(1 \mathrm{H}, 2 \mathrm{~S},-\mathrm{CHO}) \mathrm{ppm}$. 核磁数值表明, 7 系异构混合物, 它的稳定性较差, 不宜久置. 7 和 1-丁烯-3-酮反应 ${ }^{[6]}$ 生成 1.5-双酮 8, IR: $\nu_{\max } 1730$ (酯), 1710 (肩、酩). 上述两步的产率均可达 $90 \%$. 薄板层析和气相色谱均表明 产物的纯度较好.

8 用氢氧化钠一乙醇一水处理 ${ }^{[7]}$ 产生 $\alpha, \beta$-不饱和酮酸盐 9.9 用酸酸化, 再经层析生成 10 和 11 的混合物, 产率约 $25 \%$, 以 10 为主. 用丙酮一正己烷重结晶得失碳倍半萜内酯 10 , 它 和用酸处理青蓄素所得的降解产物 ${ }^{[1]}$ 相比较, 熔点、旋光事、红外、核磁均相同, 混合熔点不降 低,因而 10 的结构被确认. 当 8 用氢氧化钡一乙醇一水处理亦得 10 和 11 的混合物, 产率约 $20 \%$, 以 11 为主. 用丙酮一乙醚重结晶得化合物 11 . 化合物 10 和 11 的物理数据列于表 1 .

表 1 化合物 10 和 11 的物理常数和谱学数据

\begin{tabular}{|c|c|c|c|c|}
\hline 化 合 物 & 熔 点 ${ }^{9} \mathrm{C}$ & 红 外 $\mathrm{cm}^{-1}$ & 核 碰 ppm & 质 徣 $\mathrm{m} / \mathrm{e}$ \\
\hline 10 & $143-145$ & $\begin{array}{l}1707 \\
1767\end{array}$ & $\begin{array}{l}1.0+\left(\mathrm{C}_{10}-\mathrm{CH}_{3}\right) \\
1.23\left(\mathrm{C}_{11}-\mathrm{CH}_{3}\right) \\
2.67\left(\mathrm{C}_{11}-\mathrm{H}\right)\end{array}$ & $237\left(M^{+}+1\right)$ \\
\hline 11 & $93-95$ & $\begin{array}{l}1705 \\
1767\end{array}$ & $\begin{array}{l}1.02\left(\mathrm{C}_{10}-\mathrm{CH}_{3}\right) \\
1.16\left(\mathrm{C}_{11}-\mathrm{CH}_{3}\right) \\
3.05\left(\mathrm{C}_{11}-\mathrm{H}\right)\end{array}$ & $236\left(M^{+}\right)$ \\
\hline
\end{tabular}

由表 1 可以看出, 10 和 11 的结构相同. 但在旋光色散中两者的 Cotton 效应却相反, 如 图 2 所示. 10 呈现正的 Cotton 效应, 11 呈现负的 Cotton 效应. 根据类似的实例 ${ }^{[8]}$ 可以推定 两者环的结合方式不同, 10 的 $\mathrm{A} / \mathrm{B}$ 是顺式结合, 11 是反式结合, 如图 2 所示.

运用八区律可以看到, 10 的 $\mathrm{C}_{6}$-取代基处于左上方, 应产生正的贡献，而 11 的 $\mathrm{C}_{6}$-取代基 处于右上方,应产生负的贡献，见图 3. 因此,八区律的推论与实验的结果亦是一致的.

在应用八区律时, 我们亦考虑到当酠基的 $\beta$-位上是竖键取代基时存在反八区律的可 能性. 从分子模型来看, 我们认为利用核磁双共振技术可就 10 和 11 的构型作进一步的验 证.

当照射 $\mathrm{C}_{11}-\mathrm{H}$ 时,化合物 10 和 11 的 $\mathrm{C}_{5}-\mathrm{He}$ 分别有 $4.1 \%$ 和 $16.4 \%$ 的增值. 在 10 中, 由 于 $\mathrm{C}_{5}-\mathrm{He}_{\mathrm{e}}$ 和其它质子蜂相互有交盖, 从而使增值有偏低现象. 当照射 $\mathrm{C}_{7}-\mathrm{H}$ 时, 11 的 $\mathrm{C}_{5}-\mathrm{He}$ 有 $13.9 \%$ 的增值, 而 10 的 $\mathrm{C}_{5}-\mathrm{He}$ 却没有增值, 见图 4. 从而确证 10 为反式内酯, 11 为顺式 内酯. 去偶实验又表明, 化合物 10 和 11 中 $\mathrm{G}_{7}-\mathrm{H}$ 和 $\mathrm{C}_{11}-\mathrm{H}$ 的偶合常数分别为 12 和 6 赫, 结 合上述的 NOE 效应, 提示 10 和 11 的 $\mathrm{C}_{11}$-甲基应分别为 $\alpha$-和 $\beta$-构型. 显然, 化合物 10 中, 就 $\mathrm{C}_{11}$-甲基以及内酯环的构型而言, 本文推导的和文献 [1]报道的结果是不同的. 对于这类内 酯环的构型问题现正在作进一步的研究.

酸处理 9 时未环合的油状物经放置析离了一个固体酸 $12, \mathrm{~m} . \mathrm{p}, 171-173^{\circ} \mathrm{C}$ IR: $\nu_{\max }$

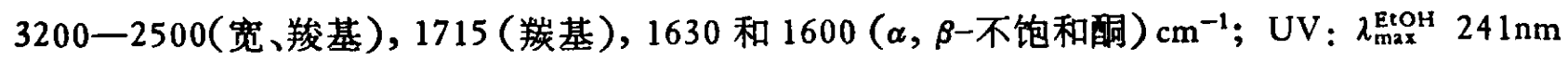
$(\in 17400)$. 用三乙基氟硼盐 $\left(\mathrm{Et}_{3} \mathrm{OBF}_{4}\right)$ 处理化合物 10、11 和 12 分别得到化合物 $13 、 14$ 和 15. 它们在旋光色散中均有相似的正的 Cotton 效应,但振幅有差异, 见图 5. 因为在双环的 $\alpha$, $\beta$-不饱和酮的体系中, 角取代基的构型对于 Cotton 效应的符号起决定的作用 ${ }^{[82 !}$, 所以它们的 $\mathrm{C}_{1}-\mathrm{H}$ 的构型应相同,均为 $\boldsymbol{\alpha}-$ 构型. 12 不能内酯环合, 可能是由于异丙基酯是坚键之故. 


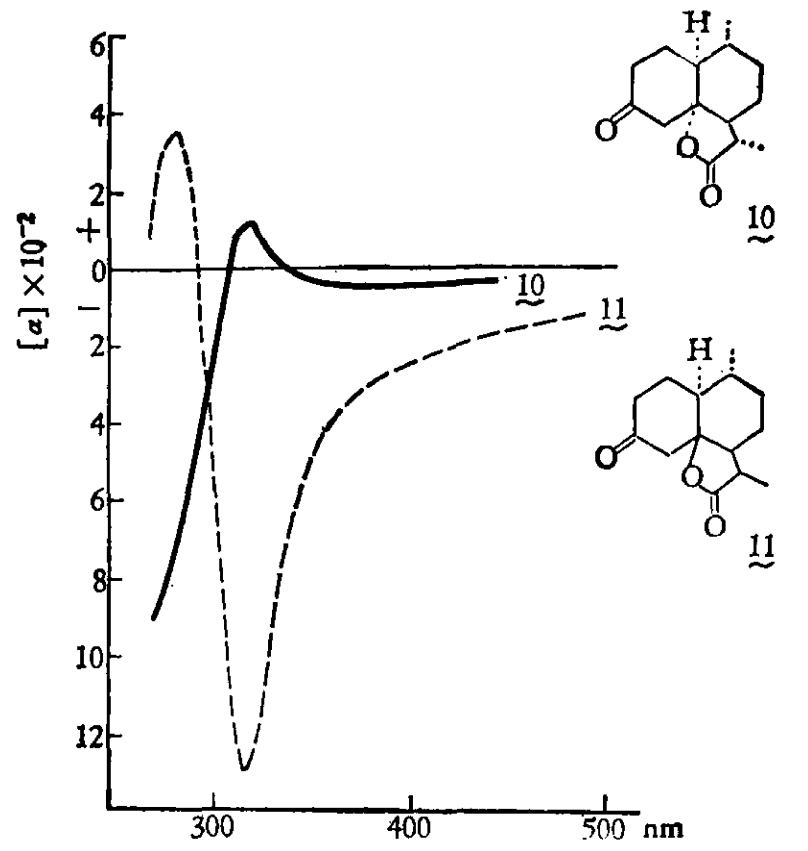

图 210 和 11 的旋光色散图谱

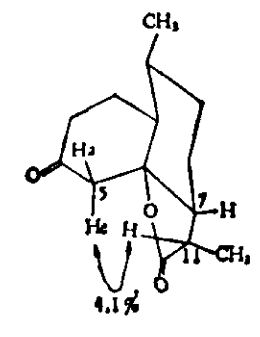

10

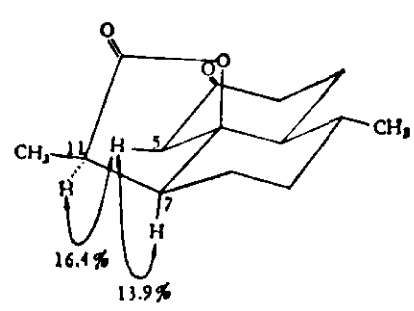

11
图 410 和 11 的 NOE 效应

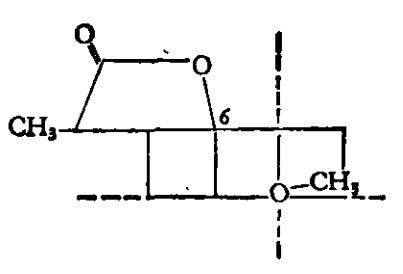

10
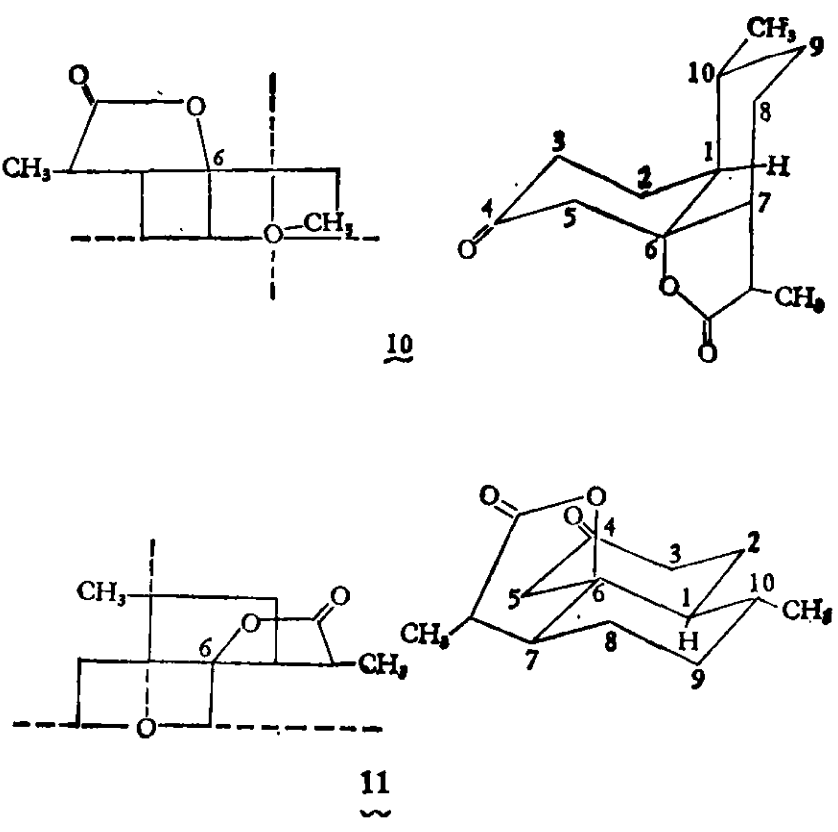

图 310 和 11 的八区律后分区简式及构象

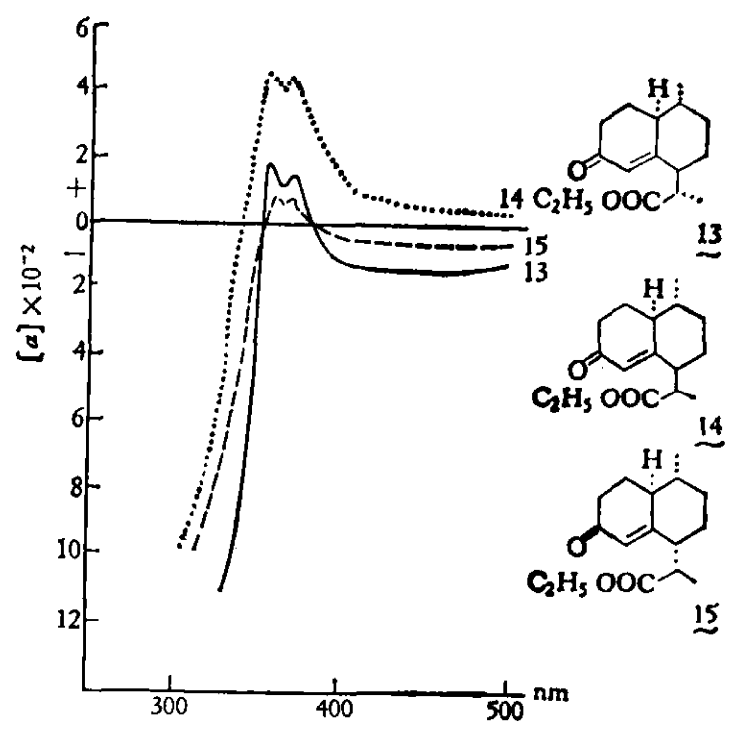

图 $513 、 14$ 和 15 的旋光色散图谱

致谢: 中国科学院上海药物研究所沈春铜同志测定了 ORD 谱, 并就图谱解释提供了宝贯的意见, 特此 票谢。

\section{参考文 嗝}

[1] 刘静明、倪募云、樊菊芬、屠地场、吴照华、吴统林、周维善，化学学报，37(1979)，129.

[2] Nakztani, T. \& Kawashima, K., Synthesis, 1978, 147.

[ 3 ] Sohulte-Elte, K. H. \& Ohloff, G., Helv. Chim. Acta, 50(1967), 153.

[ 4 ] Foote, C. S., Wuesthoft, M. T. \& Urstain, I. G. B., Tetrahedron, 23(1967), 2601.

[5] Spenceer, T. A., Weaver, T. D., Villavica, R. M., Frinary, R. J., Posler, J. \& Schwartz, M. A., J. Org. Chem., 33(1968), 712.

[6] Turner, R. B., Nettlelou, Jr. D. E. \& Ferebee, R., J. Amer. Chem. Soc, 78(1956), 5923.

[ 7 ] Barkley, L. B., Knowles, W. S., Reffelson, H. \& Thompson, Q. D., ibid., 78(1956), 411.

[ 8 ] a. 黄鸣龙, 旋光橧在有机化学中的应用, 上海科学技术出版社, 1963.

b. Djerassi, C., Closson, W., J. Amer. Chem. Soc., 78(1956), 3761; Prelog, V., Smith, H. Kh, Helo. Chim. Acta, 42(1959), 2624. 